\title{
Photon-stimulated desorption from chlorinated Si(111): Etching of SiCl by picosecond-pulsed laser irradiation
}

\author{
Tomoyuki Kirimura, ${ }^{1,2, *}$ Ken-ichi Shudo,,${ }^{1,2}$, Yujiro Hayashi, ${ }^{2}$ Yoshihito Tanaka, ${ }^{2}$ Tetsuya Ishikawa, ${ }^{2}$ and Masatoshi Tanaka ${ }^{1}$ \\ ${ }^{1}$ Department of Physics, Faculty of Engineering, Yokohama National University, 79-5 Tokiwadai, Hodogaya-ku, \\ Yokohama 240-8501, Japan \\ ${ }^{2}$ RIKEN SPring-8 Center, 1-1-1, Kouto, Sayo-cho, Sayo-gun, Hyogo 679-5148, Japan \\ (Received 11 August 2005; revised manuscript received 26 October 2005; published 9 February 2006)
}

\begin{abstract}
$\mathrm{Cl}$ etching of silicon surfaces assisted with short pulsed laser irradiation is analyzed to find out chloride removal processes without using thermal reaction. When a $\mathrm{Cl}$-saturated $\mathrm{Si}(111)$ surface was irradiated by a picosecond pulsed laser at $400 \mathrm{~nm}$, desorptions of $\mathrm{SiCl}$ and $\mathrm{SiCl}_{2}$ molecules were observed with a quadrupole mass spectrometer with a pulse-counting system. The presence of the $\mathrm{SiCl}$ desorption shows previously unfound etching process without thermally induced recombinative reaction. Time-of-flight and signal-ratio of the $\mathrm{SiCl}$ and $\mathrm{SiCl}_{2}$ molecules indicated that they were assigned to products from restatom monochlorides and adatom polychlorides, respectively. High desorption rates of the chlorides which depend on laser fluence nonlinearly suggest that multiple excitation by substrate hot carriers is essential to the etching.
\end{abstract}

DOI: 10.1103/PhysRevB.73.085309

PACS number(s): 68.43.Tj, 68.43.Vx, 81.65.Cf, 68.47.Fg

\section{INTRODUCTION}

Etching of silicon surfaces with halogen gasses is prevalent dry processing in semiconductor fabrication. A deeper understanding of elementary processes of the surface reaction is essential to realize layer-by-layer surface modification. Hence, etching pathways of the halogenated silicon surfaces have been investigated by analyzing desorbed species during thermal treatment and photon irradiation. ${ }^{1-7}$ In the thermally activated etching of chlorine-(Cl-) adsorbed $\mathrm{Si}(111)$ surface, the dominant component of the desorption is $\mathrm{SiCl}_{2}$ molecule. Monochlorides on the surface recombine with other $\mathrm{Cl}$ atoms and are subsequently desorbed as volatile dichlorides, which is attributed to weaker bonds of dichlorides to substrate $(2.8 \mathrm{eV})$ than monochlorides $(4.2$ eV). ${ }^{8}$ In contrast to the well-studied thermal reaction, elementary processes of photon-stimulated etching are ambiguously understood because of complexity arising from thermal effects of the irradiation. We here show that shorter pulsed laser irradiation to avoid the thermal effects can desorb $\mathrm{SiCl}$ molecules. This desorbed product is thermally inactive. The photon irradiation expands potential to produce thermally unachievable surface configurations.

A Cl-adsorbed $\mathrm{Si}(111)-7 \times 7$ surface is a model system for the study of site-dependent process because of the variety of adsorption sites. The clean $\mathrm{Si}(111)-7 \times 7$ surface at room temperature is composed of dimer-adatom-stacking faults. A unit cell includes 12 adatoms and 42 restatoms having dangling-bonds for $\mathrm{Cl}$ atoms to bond to. X-ray photoemission spectroscopy and scanning tunneling microscopy (STM) have revealed that the $\mathrm{Cl}$-adsorption processes depend on coverage. A lower $\mathrm{Cl}$ coverage forms monochlorides both for ad-rest-atoms ${ }^{9-11}$ whereas a higher $\mathrm{Cl}$ coverage forms adatom polychlorides due to backbond breakings and restatom monochlorides. ${ }^{12}$ At the saturation coverage, 12 adatom polychlorides and 30 restatom monochlorides are present in the unit cell. In spite of the two adsorption components, desorbed species are only $\mathrm{SiCl}_{2}$ molecules when the surface is thermally annealed, demonstrated by temperature programmed desorption (TPD). ${ }^{2,3}$

Pulsed laser-induced desorption from a Cl-adsorbed $\mathrm{Si}(111)$ surface has been investigated with nanosecond pulsed lasers to clarify etching processes different from thermal effects. The adatom polychlorides are ejected as $\mathrm{SiCl}_{2}$ molecules without diffusion ${ }^{4-6}$ whereas the restatom monochlorides are desorbed as $\mathrm{SiCl}_{n}(n=2$ to 4$)$ after the recombinative reaction between $\mathrm{SiCl}$ and $\mathrm{Cl}$ atoms. ${ }^{7}$ The desorption mechanism was concluded to be laser-induced thermal desorption (LITD) based on the results of time-of-flight (TOF) analysis and laser fluence dependence. ${ }^{13}$

Irradiations of femtosecond and picosecond pulsed lasers will be more effective ways to observe the halogen etching excluding thermal effects. This paper reports desorption of silicon chlorides from a Cl-saturated $\mathrm{Si}(111)$ surface by the irradiation of 2 ps pulsed laser at the wavelength of $400 \mathrm{~nm}$. The compositions of the ejected species as well as the laser fluence dependence are significantly different from those of the nanosecond pulsed laser excitation. Through measurements of TOF and quantitative analysis of the desorbed species, we first found desorption of $\mathrm{SiCl}$ molecules from this surface by electronic excitation. The desorption rate is much higher than that from the bare $\mathrm{Si}(111)$ surface.

\section{EXPERIMENT}

Neutral particles ejected from a Cl-saturated $\mathrm{Si}(111)$ surface by laser irradiation were observed with quadrupole mass spectrometer (QMS, Spectra International: Windows Satellite 300D). Cracking of the neutral particles caused by ionizations, e.g., $\mathrm{SiCl}_{2} \longrightarrow \mathrm{SiCl}^{+}+\mathrm{Cl}^{+}$, is an obstructive problem in chemical composition analysis with the QMS. In order to identify actual yield by desorbed species, we examined the cracking contribution using a TOF technique.

A plate $\left(7 \times 20 \times 0.38 \mathrm{~mm}^{3}\right)$ of boron-doped $p$-type $(10 \Omega \mathrm{cm}) \mathrm{Si}(111)$ wafer was set in an ultrahigh vacuum of $2 \times 10^{-8} \mathrm{~Pa}$. The $\mathrm{Si}(111)$ surface was cleaned by $\mathrm{Ar}^{+}$sputter- 
ing and Ohmic heating up to $1420 \mathrm{~K}$, and $7 \times 7$ structure of the clean surface was confirmed by low-energy electron diffraction (LEED). The surface was exposed to a $\mathrm{Cl}$ gas supplied from electrolysis cell of $\mathrm{AgCl}$ pellet. A QMS ionizer was placed $45 \mathrm{~mm}$ from the surface. All the measurements were performed on the $\mathrm{Cl}$-saturated surface at room temperature.

Laser system was composed of a mode-locked Ti:sapphire oscillator [Spectra-Physics Lasers (SP), Tsunami] and a regenerative amplifier (SP, Spitfire). A laser beam produced at the oscillator with the wavelength of $800 \mathrm{~nm}$ was guided to the regenerative amplifier. The output beam from the regenerative amplifier had the pulse duration of $2 \mathrm{ps}$ [full width at half maximium (FWHM)] with the repetition rate of $1 \mathrm{kHz}$. The laser beam at the wavelength of $400 \mathrm{~nm}$ was provided by using $\beta$-barium borate (BBO) crystal. An incident angle was set at $45^{\circ}$ to the surface normal. The spot size of the beam on the surface was $0.44 \mathrm{~mm}^{2}$. A timing controlled shutter system was used to pick up a single shot from the $1 \mathrm{kHz}$ train of the laser pulse at an appropriate timing for data acquisition.

A QMS was equipped with a high-gain multiplier operating in pulse-counting mode. ${ }^{15}$ Output pulses via fast preamplifier (EG\&G ORTEC9306) and discriminator (Philips Scientific: MODEL708) were accumulated with multichannelscaler (MCS, ORTEC: 100-ps Time digitizer/MCS Model 9353) for TOF measurements or counter (EG\&G ORTEC994) for TPD measurements. The MCS was triggered by incident laser pulses which were detected by a photodiode. A drift time caused by acceleration of ions produced at an ionizer toward a detector was corrected in TOF spectrum. ${ }^{16}$ In the TPD, signals for $\mathrm{SiCl}^{+}, \mathrm{SiCl}_{2}{ }^{+}, \mathrm{SiCl}_{3}{ }^{+}$, and $\mathrm{SiCl}_{4}{ }^{+}$were counted in turn for each integration time of $0.2 \mathrm{~s}$ with temperature ramp of $2 \mathrm{~K} / \mathrm{s}$. The temperature was monitored by a radiation thermometer.

\section{RESULTS AND DISCUSSION}

Desorption signals under laser irradiation were found at the masses of $\mathrm{SiCl}^{+}(m / e=63), \mathrm{SiCl}_{2}{ }^{+}(m / e=98)$, and $\mathrm{Si}^{+}(m / e=28) . \mathrm{SiCl}_{3}{ }^{+}, \mathrm{SiCl}_{4}{ }^{+}$, and other signals were negligibly small. When a clean $\mathrm{Si}(111)-7 \times 7$ surface was irradiated by the laser, the $\mathrm{Si}^{+}$signal was below our detection limit. These indicate that a $\mathrm{Cl}$-adsorption promotes laserinduced desorption of surface adsorbates without that of substrate silicon atoms.

\section{A. Time of flight spectra}

Figure 1 shows the TOF spectra for ${ }^{*} \mathrm{SiCl}^{+}, \mathrm{SiCl}_{2}{ }^{+}$, and $\mathrm{Si}^{+}$at the laser fluence of $10 \mathrm{~mJ} / \mathrm{cm}^{2}$. The spectra were accumulated for 200 scans within which desorption of the chlorides finished.

It has already been demonstrated that $\mathrm{SiCl}_{2}$ molecules are cracked into $\mathrm{SiCl}^{+}$and $\mathrm{SiCl}_{2}{ }^{+}$ions by ionization. The ratio of $\mathrm{SiCl}^{+} / \mathrm{SiCl}_{2}{ }^{+}$was 3.8 in our experiment as confirmed from TPD measurement afterward. This value approximately agrees with the results of other studies. ${ }^{2,4} \mathrm{In}$ the ${ }^{*} \mathrm{SiCl}^{+}$spectrum, the $3.8 \times \mathrm{SiCl}_{2}{ }^{+}$spectrum was subtracted to remove

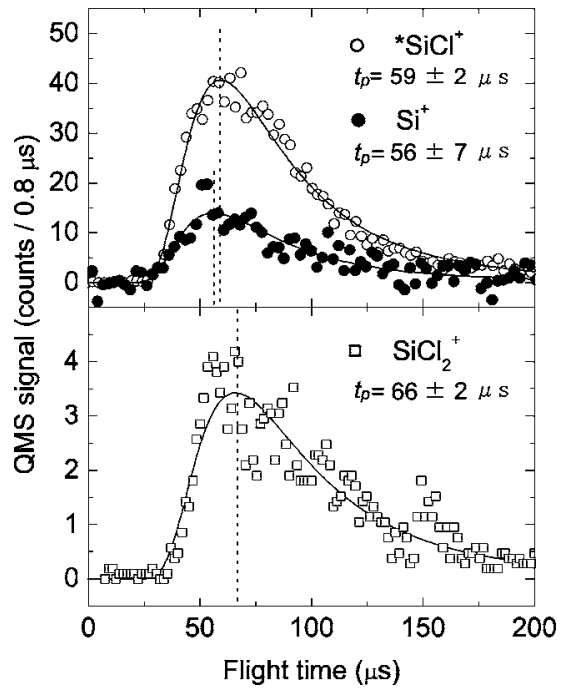

FIG. 1. TOF spectra for ${ }^{*} \mathrm{SiCl}^{+}$(open circles), $\mathrm{Si}^{+}$(dots), and $\mathrm{SiCl}_{2}{ }^{+}$(open squares) by $10 \mathrm{~mJ} / \mathrm{cm}^{2}$ laser irradiation. Accumulative number was 200 for which the desorption finished completely. The ${ }^{*} \mathrm{SiCl}^{+}$spectrum corresponds to $\mathrm{SiCl}^{+}$spectrum subtracted by $3.8 \times \mathrm{SiCl}_{2}{ }^{+}$spectrum which is the contribution from cracking of desorbed $\mathrm{SiCl}_{2}$ molecules. Average noise level due to $\mathrm{CO}^{+}$is corrected in the $\mathrm{Si}^{+}$spectrum. The thin curves are fitted with modified Maxwell-Boltzmann distribution. $t_{p}$ is peak time of each spectrum.

$\mathrm{SiCl}^{+}$signal originating from $\mathrm{SiCl}_{2}$ molecules, i.e., the spectrum represents the distribution of desorbed $\mathrm{SiCl}$ molecules. The $\mathrm{SiCl}_{2}{ }^{+}$spectrum is the distribution of desorbed $\mathrm{SiCl}_{2}$ molecules. For $\mathrm{Si}^{+}$spectrum, noise level contributed by a residual gas of $\mathrm{CO}^{+}(m / e=28)$ was subtracted.

The spectra are fitted well with a modified MaxwellBoltzmann distribution (solid lines in Fig. 1), ${ }^{17}$

$$
f(t) d t=\frac{a}{t^{4}} \exp \left\{-b\left(\frac{z}{t}-c\right)^{2}\right\} d t,
$$

where $z$ is a flight distance, $t$ is a flight time, and $a, b$, and $c$ are fitting parameters. From the fittings, we determined the parameters giving the profile properly. The $t_{p}$ in Fig. 1 is a peak time of the TOF profile calculated from the parameters. The peak times between $\mathrm{SiCl}^{+}$and $\mathrm{Si}^{+}$coincide within the margin of error while those between $\mathrm{SiCl}^{+}$and $\mathrm{SiCl}_{2}{ }^{+}$are quite distinct. The large margin of error for $\mathrm{Si}^{+}$arises from the contribution of $\mathrm{CO}^{+}$. The similarity between the $\mathrm{SiCl}^{+}$ and $\mathrm{Si}^{+}$profiles shows that they both originate from $\mathrm{SiCl}$ molecules. On the other hand, the difference between the $\mathrm{SiCl}^{+}$and $\mathrm{SiCl}_{2}{ }^{+}$indicates that $\mathrm{SiCl}$ and $\mathrm{SiCl}_{2}$ molecules are primarily desorbed species. From integrations of the TOF spectra, the cracking ratio of the $\mathrm{SiCl}^{+} / \mathrm{Si}^{+}$from $\mathrm{SiCl}$ molecules was 2.4.

\section{B. Translational temperature and surface temperature}

We calculated translational energies of desorbed $\mathrm{SiCl}$ and $\mathrm{SiCl}_{2}$ molecules from TOF spectra at room temperature and liquid nitrogen temperature to estimate whether observed desorption was thermally induced or not. Note that the TOF spectra in Fig. 1 do not correspond to flux distributions of 
desorbed particles. Since high velocity neutral particles are less frequently ionized than low velocity ones due to the electron impact at ionizer, QMS intensity is inversely proportional to the velocity. ${ }^{17}$ Therefore, a mean translational energy $\left\langle E_{t}\right\rangle$ obtained from Fig. 1 is given by

$$
\left\langle E_{t}\right\rangle=\int E_{t} f(t) t^{-1} d t \iint f(t) t^{-1} d t,
$$

where $f(t)$ is the observed distribution and $f(t) t^{-1}$ is proportional to the flux distribution. Translational temperatures of $\left\langle E_{t}\right\rangle / 2 k_{B}$ for the $\mathrm{SiCl}^{+}$and $\mathrm{SiCl}_{2}{ }^{+}$spectra were turned out to be 1160 and $1410 \mathrm{~K}$, respectively, which were constant in the fluence range of $4-15 \mathrm{~mJ} / \mathrm{cm}^{2}$. The difference in the translational temperatures will be ascribable to that in potential barriers for desorption of monochlorides and dichlorides on the surface because of their different etching energies. ${ }^{8}$ Surface temperature does not influence the mean translational temperatures because the TOF spectra for a cooled surface at liquid nitrogen temperature were the same as those at room temperature. It is also necessary to estimate a temperature rise of the surface due to a single shot for discussion on the thermal effect. The temperature rise was calculated with the solution of heat conduction equation by Bechtel. ${ }^{18}$ The maximum surface temperature for the fluence $10 \mathrm{~mJ} / \mathrm{cm}^{2}$ turned out to be about $930 \mathrm{~K}$ on a time scale of picoseconds. The thermal effect is not the essence of the desorption of $\mathrm{SiCl}$ molecules because it is never observable at $900 \mathrm{~K}$ even on a long time scale of seconds as is clear from TPD and isothermal measurements. These results would show that desorption processes of $\mathrm{SiCl}$ and $\mathrm{SiCl}_{2}$ molecules by the laser irradiation are not related to thermal equilibrium but electronically nonequilibrium condition.

\section{Desorption cross section}

A crucial difference between picosecond and nanosecond pulsed laser induced etchings was found in desorption cross sections. We measured desorption yield of $\mathrm{SiCl}^{+}$and $\mathrm{SiCl}_{2}{ }^{+}$ per a laser shot at the fluence of $10 \mathrm{~mJ} / \mathrm{cm}^{2}$ and plotted it with respect to the number of a sequence of incident laser shots in Fig. 2. The yield is largest at the first shot and decreases very fast during ten shots. The desorption cross section $\sigma_{d}$ is obtained from the downward curve. When $\theta_{x}$ corresponds to surface coverage before the $x$ th laser shot, the change in the surface coverage $-d \theta_{x} / d x$ is given by

$$
-d \theta_{x} / d x=\theta_{x} I \sigma_{d},
$$

where $I$ is the number of incident photons per unit area. Desorption yield $Y(x)$ due to the $x$ th laser shot is proportional to $-d \theta_{x} / d x . Y(x)$ is therefore expressed with an exponential term, $Y(x)=A \exp \left(-I \sigma_{d} x\right)$, where $A$ is a constant. The curves of Fig. 2 are fitted well with linear combination of two exponential functions $Y(x)=A_{1} \exp \left(-I \sigma_{d 1} x\right)+A_{2} \exp \left(-I \sigma_{d 2} x\right)$, which are drawn by solid lines. The subscripts 1 and 2 represent the components of fast and slow decays, respectively. The fitting parameters of $\sigma_{d 1}$ and $\sigma_{d 2}$ were $5.4 \times 10^{-17}$ and $2.7 \times 10^{-18} \mathrm{~cm}^{2}$, respectively. For $\mathrm{SiCl}^{+}$and $\mathrm{SiCl}_{2}{ }^{+}$, the cross sections were approximately equal. Compared with a result

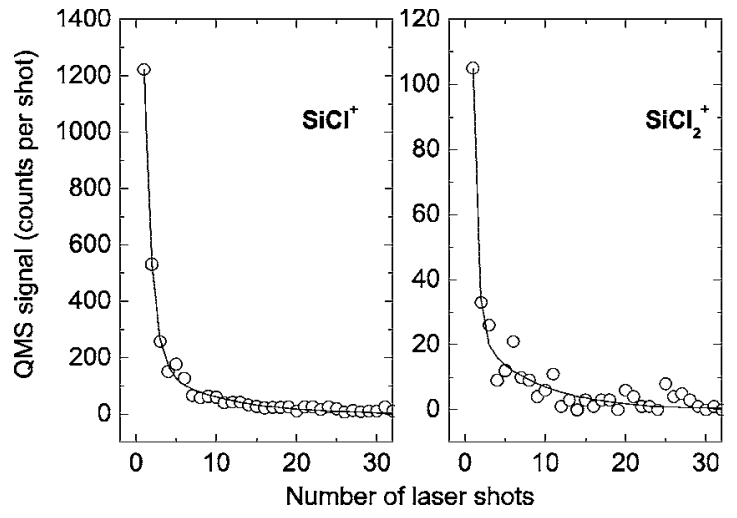

FIG. 2. Desorption yield for $\mathrm{SiCl}^{+}$and $\mathrm{SiCl}_{2}{ }^{+}$with respect to a sequence of the laser irradiation at the fluence of $10 \mathrm{~mJ} / \mathrm{cm}^{2}$ (open circles). The solid lines are fitting curves with linear combination of two exponential functions of fast and slow decays.

for nanosecond pulsed laser irradiation, ${ }^{6}$ the cross sections are larger by more than a factor of $10^{2}$. This concludes that shorter pulse duration affects the enhancement of etch rate.

The fitting also reflects that two (or more) desorption cross sections exist. We can deduce that the cross sections of fast and slow decays are dependent on $\mathrm{Cl}$ coverage. According to cluster calculation, ${ }^{19}$ higher $\mathrm{Cl}$ concentration makes weaker bonds of chlorides to substrate. In addition, the study of Aizawa et al. showed that reduction of the bond energies is due to surface strain which is caused by an interatomic repulsion between halogens. ${ }^{20}$ Therefore, the fast decay presumably corresponds to desorptions from high $\mathrm{Cl}$ concentration sites such as polychlorides and their adjacent monochlorides at high coverage. The slow decay would be desorption from monochlorides at low coverage after major desorption during first several laser shots.

To identify the desorption sites from observation of surface adsorbates after laser irradiation, TPD is a useful method. The Solid lines in Fig. 3 are TPD spectra of a Clsaturated $\mathrm{Si}(111)$ surface. The resemblance between spectral profiles of the $\mathrm{SiCl}^{+}$and $\mathrm{SiCl}_{2}^{+}$, whose intensity ratio is $3.8: 1$, shows that the $\mathrm{SiCl}_{2}$ molecules are dominantly desorbed in the thermal process. ${ }^{1-3}$ Desorption stages corresponding to three peaks in $\mathrm{SiCl}^{+}$and $\mathrm{SiCl}_{2}{ }^{+}$spectra have been studied well by STM, ${ }^{10,11,21}$ isothermal and TPD measurements with QMS, ${ }^{1-3}$ and surface differential reflectivity spectroscopy. ${ }^{22}$ Their studies have shown that the desorbed $\mathrm{SiCl}_{2}$ molecules originate from surface components of adatom polychlorides and restatom monochlorides although recombinative reaction is involved in the process. Thus, yields of the $\mathrm{SiCl}^{+}$and $\mathrm{SiCl}_{2}{ }^{+}$are relative amounts of the surface components. The dashed lines in Fig. 3 are the TPD spectra after 10 shots at the fluence of $10 \mathrm{~mJ} / \mathrm{cm}^{2}$. The surface was irradiated over one third of the total surface area by displacing substrate position. After the irradiation, the intensities uniformly decreased by approximately the same ratio of one third. This shows that the surface components of adatom polychlorides and restatom monochlorides were diminished after the 10 shots. The decrease was not observed at low laser fluence where nothing is desorbed. Previous studies on photon-stimulated desorption from a semiconductor 


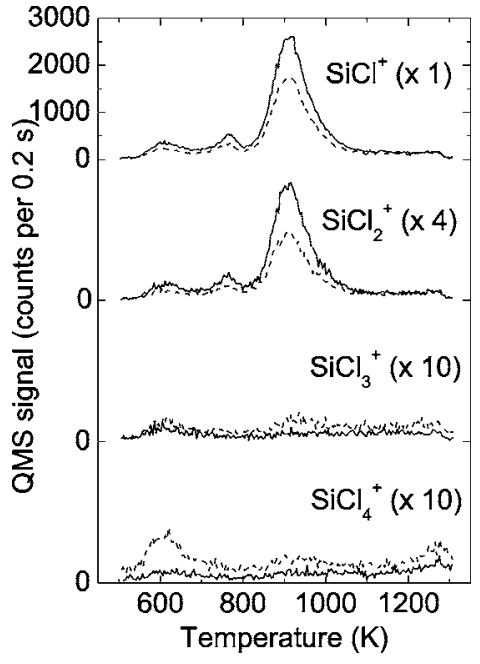

FIG. 3. TPD spectra of the Cl-saturated $\mathrm{Si}(111)$ surface (solid lines) and those after 10 laser shots at the fluence of $10 \mathrm{~mJ} / \mathrm{cm}^{2}$ (dashed lines). The irradiated area was nearly one-third of the surface. The rate of temperature rise was $2 \mathrm{~K} / \mathrm{s}$. $\mathrm{SiCl}^{+}$is cracking products from $\mathrm{SiCl}_{2}$ molecules, and the ratio of $\mathrm{SiCl}^{+} / \mathrm{SiCl}_{2}{ }^{+}$is $\sim 3.8$.

surface $^{23}$ and a metal surface ${ }^{24}$ concluded that fast decay component as shown in Fig. 2 corresponds to desorption from surface defect sites. However, the TPD revealed that the fast decay we observed is from the surface components.

\section{Desorbed species and the original adsorption sites}

It is proved above that pathway from photoabsorption to etching will not contain recombinative reaction as seen in thermal desorption. The evidence is threefold: TOF spectra are independent on the surface temperature, desorption yield is very large even in a single shot, and thermal desorption of $\mathrm{SiCl}$ molecule is not observed in TPD. In fact, the study for desorption induced by nanosecond pulsed UV laser by Hattori et al. suggested that $\mathrm{SiCl}_{2}$ molecule is desorbed from adatom polychlorides without recombinative reaction. ${ }^{4}$ The calculation of etching energy by Sakurai et al. also supports that the adatom polychlorides prefer to be ejected as $\mathrm{SiCl}_{2}$ molecules. ${ }^{8}$ Their reports and our result that $\mathrm{SiCl}$ molecules are desorbed can provide a picture that the $\mathrm{SiCl}_{2}$ molecule is derived from adatom polychloride and the $\mathrm{SiCl}$ molecule is derived from restatom monochloride.

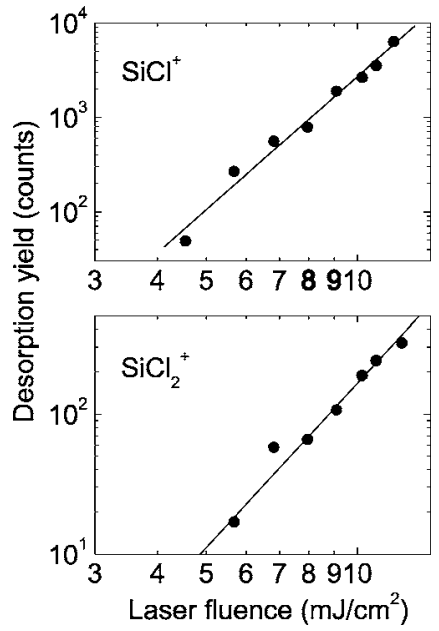

FIG. 4. Laser fluence dependences of the desorption yield for $\mathrm{SiCl}^{+}$and $\mathrm{SiCl}_{2}{ }^{+}$. Fittings with power function are shown in solid lines. The power law factors are 4.7 and 3.9 for the $\mathrm{SiCl}^{+}$and $\mathrm{SiCl}_{2}{ }^{+}$, respectively.

Numerical integration values for TOF spectra indicate that the amount of $\mathrm{SiCl}^{+}$is approximately ten times larger than that of $\mathrm{SiCl}_{2}{ }^{+}$. This quantitative difference can be interpreted by the picture. In Table I, we simulated intensity ratio of detected $\mathrm{SiCl}^{+}$and $\mathrm{SiCl}_{2}{ }^{+}$which are produced by $\mathrm{SiCl}$ molecules ejected from 30 restatom monochlorides and by $\mathrm{SiCl}_{2}$ molecules ejected from 12 adatom polychlorides in a unit cell at saturation coverage. Then the cracking rates of the $\mathrm{SiCl}$ and $\mathrm{SiCl}_{2}$ molecules were considered. In consequence, the estimated ratio is close to $10: 1$ and in good agreement with the experimental result. This supports that the $\mathrm{SiCl}$ molecules and the $\mathrm{SiCl}_{2}$ molecules are derived from the restatom monochlorides and adatom polychlorides, respectively.

\section{E. Mechanism of photoassisted etching}

The electronic process induced by picosecond pulsed laser would be distinct from that by nanosecond pulsed laser. It was shown from laser fluence dependences. Double logarithmic plots of yields of $\mathrm{SiCl}^{+}$and $\mathrm{SiCl}_{2}{ }^{+}$with respect to the laser fluence are shown in Fig. 4. The yields are integrations of TOF spectra at each fluence. Solid lines are fittings with a power function $Y=a F^{b}$. They indicate superlinear dependences with power law factors $b$ of 4.7 and 3.9, respectively,

TABLE I. Simulated QMS signals under the assumption that restatom monochlorides are desorbed as $\mathrm{SiCl}$ molecules and adatom polychlorides are desorbed as $\mathrm{SiCl}_{2}$ molecules from a unit cell. The cracking rates of the $\mathrm{SiCl}$ and $\mathrm{SiCl}_{2}$ molecules are obtained from the results of TOF and TPD measurements. The number ratio between the total $\mathrm{SiCl}^{+}$and $\mathrm{SiCl}_{2}{ }^{+}$is about $10: 1$.

\begin{tabular}{lccc}
\hline \hline & $\begin{array}{c}\text { Restatom monochlorides } \\
(30 / \text { unit cell }) \rightarrow \mathrm{SiCl}\end{array}$ & $\begin{array}{c}\text { Adatom polychlorides } \\
(12 / \text { unit cell }) \rightarrow \mathrm{SiCl}_{2}\end{array}$ & Total \\
\hline Cracking rate & $\mathrm{SiCl} \rightarrow \mathrm{SiCl}^{+} / \mathrm{Si}^{+}=2.4$ & $\mathrm{SiCl}_{2} \rightarrow \mathrm{SiCl}^{+} / \mathrm{SiCl}_{2}{ }^{+}=3.8$ & \\
$\mathrm{SiCl}^{+}$ & 21 & 9 & 30 \\
$\mathrm{SiCl}_{2}{ }^{+}$ & & 3 & 3 \\
$\mathrm{Si}^{+}$ & 9 & & 9 \\
\hline \hline
\end{tabular}


which presents that the desorption is a high order reaction. This is quite different from the results by nanosecond pulsed lasers in which yield increases linearly with the fluence. ${ }^{13,25}$ Chen et al. observed linear fluence dependence of etching from monochlorinated adatom, studied by $193 \mathrm{~nm}$ (photon energy $6.3 \mathrm{eV}$ ) nanosecond pulsed laser irradiation. They concluded that a single-photon absorption induced etching of a single adatom. However, process due to multi-photon excitation will be required for etching of a chloride by $400 \mathrm{~nm}$ laser irradiation we used since the photon energy $(400 \mathrm{~nm}$ $=3.10 \mathrm{eV})$ is not enough to remove monochlorides and dichlorides whose bond energies are 4.2 and $2.8 \mathrm{eV}$, respectively. ${ }^{8}$

The presence of substrate hot carriers generated by photoabsorption has a strong relationship to desorption, which has been recognized on both metal and semiconductor surfaces. The notable feature is that laser irradiation with pulse duration of a time scale of electron-hole recombination increases desorption rate of surface adsorbates which nonlinearly depends on the laser fluence. ${ }^{26}$ Two possible mechanisms were suggested for the hot carriers-induced process from experimental and theoretical approaches: desorption induced by multiple electronic transitions ${ }^{14,17,26,27}$ (DIMET) and desorption due to nonadiabatic vibrational excitations. ${ }^{28-31}$ In DIMET, the adsorbates pass through an electronic excited state by photoabsorption and hot carrier scattering. When a lifetime of the excited state of the adsorbates is shorter than the time to acquire an energy needed to desorb, the desorption rate due to only a single electronic transition is low. However, as hot carrier scattering effectively prolongs the lifetime of the excited state in repeated electronic transitions, the desorption rate is significantly enhanced. In nonadiabatic vibrational excitations, the adsorbates do not pass through the electronic excited state. Energy transfer from the hot carriers to nuclear degree of freedom through nonadiabatic coupling is responsible for vibrational excitation of the surface adsorbate. Multiple vibrational excitations until unbound state lead to desorption. Pulsed laser irradiation with a time scale of hot carrier lifetime, in either mechanism, generates dense electron-hole pairs in substrate, which would enable efficient desorption.

In the case of silicon, the substrate electrons excited to a conduction band recombine with holes in a valence band during $1 \mathrm{ps}$ and reach equilibrium with a lattice. ${ }^{32,33}$ The laser pulse duration of $2 \mathrm{ps}$, we used, which is near the lifetime of the hot carriers will be important to the etchings of restatom monochlorides and adatom polychlorides, and the large desorption cross section shown in Fig. 2 would reflect the hot carrier process. The nonlinear dependence shown in Fig. 4 suggests that multiple excitation of the surface adsorbates is the crucial process of the desorption.

\section{SUMMARY}

TOF distribution and the quantitative analyses have revealed etching process of a Cl-saturated $\mathrm{Si}(111)$ surface stimulated by a picosecond pulsed laser. The pulse duration remarkably affected desorbed species and rate. Desorption of restatom monochlorides as $\mathrm{SiCl}$ molecules and that of adatom polychlorides as $\mathrm{SiCl}_{2}$ molecules were first observed from this surface. The desorptions would not involve recombinative reaction as thermal process. The large cross section and the nonlinearity of the fluence dependence suggest that the etching is related to multiple excitation caused by substrate hot carriers. These findings conclude that a short pulsed laser enables efficient atomic layer modification under small thermal effect. Laser processing of the halogenadsorbed surfaces will serve for layer-by-layer control.

\section{ACKNOWLEDGMENTS}

This research was partially supported by the Ministry of Education, Culture, Sports, Science and Technology, Grantin-Aid for Scientific Research (Young Scientists B), Grant No. 16740172 (FY2004-2005).
*Present address: RIKEN SPring-8 Center, 1-1-1, Kouto, Sayo-cho, Sayo-gun, Hyogo 679-5148, Japan.

†Electronic address: ken1@ynu.ac.jp

${ }^{1}$ T. Shirao, K. Shudo, Y. Tanaka, T. Nakajima, T. Ishikawa, and M. Tanaka, Jpn. J. Appl. Phys. 42593 (2003).

${ }^{2}$ P. Gupta, P. A. Coon, B. G. Koehler, and S. M. George, Surf. Sci. 249, 92 (1991).

${ }^{3}$ P. Gupta, P. A. Coon, B. G. Koehler, and S. M. George, J. Chem. Phys. 93, 2827 (1989).

${ }^{4}$ K. Hattori, K. Shudo, T. Iimori, F. Komori, Y. Murata, J. Phys.: Condens. Matter 8, 6543 (1996).

${ }^{5}$ K. Shudo, F. Komori, K. Hattori, and Y. Murata, Surf. Sci. 320, 161 (1994).

${ }^{6}$ T. Iimori, K. Hattori, K. Shudo, T. Iwaki, M. Ueta, and F. Komori, Appl. Surf. Sci. 130, 90 (1998).

${ }^{7}$ H. Amasuga, M. Nakamura, Y. Mera, and K. Maeda, Appl. Surf. Sci. 197, 577 (2002).
${ }^{8}$ S. Sakurai and T. Nakayama, Jpn. J. Appl. Phys. 41, 2171 (2002).

${ }^{9}$ L. J. Whitman, S. A. Joyce, J. A. Yarmoff, F. R. McFeely, and L. J. Terminello, Surf. Sci. 232, 297 (1990).

${ }^{10}$ J. S. Villarrubia and J. J. Boland, Phys. Rev. Lett. 63, 306 (1989).

${ }^{11}$ J. J. Boland and J. S. Villarrubia, Phys. Rev. B 41, 9865 (1990).

${ }^{12}$ R. D. Schnell, D. Rieger, A. Bogen, F. J. Himpsel, K. Wandelt, and W. Steinmann, Phys. Rev. B 32, 8057 (1985).

${ }^{13}$ Y.-L. Li, Z.-J. Zhang, Q.-K. Zheng, Z.-K. Jin, Z.-K. Wu, and Q.-Z. Qin, Appl. Phys. Lett. 53, 1955 (1988); Y.-L. Li, Q.-K. Zheng, Z.-K. Jin, M. Yu, Z.-K. Wu, and Q.-Z. Qin, J. Phys. Chem. 93, 5531 (1989).

${ }^{14}$ F.-J. Kao, D. G. Busch, D. Gomes da Costa, and W. Ho, Phys. Rev. Lett. 70, 4098 (1993).

${ }^{15}$ T. Nakajima, K. Shudo, T. Shirao, Y. Tanaka, T. Ishikawa, and M. Tanaka, J. Vac. Soc. Jpn. 45, 74 (2002).

${ }^{16} \mathrm{R}$. Braun and P. Hess, Int. J. Mass Spectrom. Ion Process. 125, 229 (1993). 
${ }^{17}$ F. M. Zimmermann and W. Ho, Surf. Sci. Rep. 22, 127 (1995).

${ }^{18}$ J. H. Bechtel, J. Appl. Phys. 46, 1975 (1975).

${ }^{19}$ P. V. Smith and Pei-Lin Cao, J. Phys.: Condens. Matter 7, 7125 (1995).

${ }^{20}$ H. Aizawa, S. Tsuneyuki, and T. Ogitsu, Surf. Sci. 438, 18 (1999).

${ }^{21}$ R. J. Pechman, X.-S. Wang, and J. H. Weaver, Phys. Rev. B 52, 11412 (1995).

${ }^{22}$ M. Tanaka, S. Minami, K. Shudo, and E. Yamakawa, Surf. Sci. 527, 21 (2003).

${ }^{23}$ K. Hattori, Y. Nakai, and N. Itoh, Surf. Sci. Lett. 227, L115 (1990); Y. Nakai, K. Hattori, and N. Itoh, Appl. Phys. Lett. 56 1980 (1990).

${ }^{24}$ K. Mase, K. Fukutani, and Y. Murata, J. Chem. Phys. 96, 5523 (1992).

${ }^{25}$ X. H. Chen, J. C. Polanyi, and D. Rogers, Surf. Sci. 376, 77
(1997).

${ }^{26}$ J. A. Prybyla, T. F. Heinz, J. A. Misewich, M. M. T. Loy, and J. H. Glownia, Phys. Rev. Lett. 64, 1537 (1990); J. A. Prybyla, H. W. K. Tom, and G. D. Aumiller, ibid. 68, 503 (1992).

${ }^{27}$ J. A. Misewich, T. F. Heinz, and D. M. Newns, Phys. Rev. Lett. 68, 3737 (1992).

${ }^{28}$ H. Aizawa and S. Tsuneyuki, Surf. Sci. 377, 610 (1997).

${ }^{29}$ J. W. Gadzuk, Chem. Phys. 251, 87 (2000).

${ }^{30}$ R. R. Cavanagh, D. S. King, J. C. Stephenson, and T. F. Heinz, J. Phys. Chem. 97, 786 (1993).

${ }^{31}$ M. Head-Gordon, and J. C. Tully, Phys. Rev. B 46, 1853 (1992).

${ }^{32}$ J. R. Goldman and J. A. Prybyla, Phys. Rev. Lett. 72, 1364 (1994).

${ }^{33}$ S. Jeong, H. Zacharias, and J. Bokor, Phys. Rev. B 54, R17300 (1996). 